\title{
Development and validation of a prognostic model incorporating texture analysis derived from standardised segmentation of PET in patients with oesophageal cancer
}

\author{
Kieran G Foley ${ }^{1} \cdot$ Robert K. Hills ${ }^{2} \cdot$ Beatrice Berthon $^{3} \cdot$ Christopher Marshall $^{3}$. \\ Craig Parkinson ${ }^{4}$. Wyn G. Lewis ${ }^{5}$ Tom D. L. Crosby ${ }^{6} \cdot$ Emiliano Spezi $^{4}$ • \\ Stuart Ashley Roberts ${ }^{7}$
}

Received: 7 December 2016 / Revised: 26 June 2017 / Accepted: 28 June 2017 /Published online: 2 August 2017

(C) The Author(s) 2017. This article is an open access publication

\begin{abstract}
Objectives This retrospective cohort study developed a prognostic model incorporating PET texture analysis in patients with oesophageal cancer (OC). Internal validation of the model was performed.

Methods Consecutive OC patients $(n=403)$ were chronologically separated into development $(n=302$, September 2010 September 2014, median age $=67.0$, males $=227$, adenocarcinomas $=237)$ and validation cohorts $(n=101$, September 2014-July 2015, median age $=69.0$, males $=78$, adenocarcinomas $=79$ ). Texture metrics were obtained using a machinelearning algorithm for automatic PET segmentation. A Cox regression model including age, radiological stage, treatment and 16 texture metrics was developed. Patients were stratified into quartiles according to a prognostic score derived from the
\end{abstract}

Dr. Emiliano Spezi and Dr. Ashley Roberts have contributed equally to this work and are considered joint last authors.

Electronic supplementary material The online version of this article (doi:10.1007/s00330-017-4973-y) contains supplementary material, which is available to authorized users.

Kieran G Foley

foleykg@ cardiff.ac.uk

Division of Cancer \& Genetics, Cardiff University, Cardiff, UK

2 Haematology Clinical Trials Unit, Cardiff University, Cardiff, UK

Wales Research \& Diagnostic PET Imaging Centre, Cardiff, UK

4 School of Engineering, Cardiff University, Cardiff, UK

5 Department of Upper GI Surgery, University Hospital of Wales, Cardiff, UK

6 Department of Oncology, Velindre Cancer Centre, Cardiff, UK

7 Department of Clinical Radiology, University Hospital of Wales, Cardiff, UK model. A $p$-value $<0.05$ was considered statistically significant. Primary outcome was overall survival (OS).

Results Six variables were significantly and independently associated with OS: age [HR $=1.02$ (95\% CI 1.01-1.04), $p<0.001]$, radiological stage [1.49 (1.20-1.84), $p<0.001]$, treatment [0.34 (0.24-0.47), $p<0.001], \log (\mathrm{TLG})[5.74$ (1.44-22.83), $p=0.013], \log$ (Histogram Energy) [0.27 $(0.10-0.74), p=0.011]$ and Histogram Kurtosis [1.22 (1.04$1.44), p=0.017]$. The prognostic score demonstrated significant differences in OS between quartiles in both the development $\left(X^{2} 143.14\right.$, df $\left.3, p<0.001\right)$ and validation cohorts $\left(X^{2}\right.$ 20.621, df $3, p<0.001$ ).

Conclusions This prognostic model can risk stratify patients and demonstrates the additional benefit of PET texture analysis in OC staging.

Key points

- PET texture analysis adds prognostic value to oesophageal cancer staging.

- Texture metrics are independently and significantly associated with overall survival.

- A prognostic model including texture analysis can help risk stratify patients.

Keywords Neoplasms · Oesophagus · Prognosis ·

Positron-emission tomography $\cdot$ Survival

\section{Introduction}

Medical imaging is a fundamental component of cancer staging worldwide and forms a substantial part of current prognostic stratification tools. Innovative radiological techniques are expected to have a substantial role in developing future 
risk stratification models, which may subsequently influence clinical decision-making.

Cross-sectional imaging allows three-dimensional (3D) tumour visualisation, enabling non-invasive, quantitative analysis of tumour heterogeneity [1]. Texture analysis of medical images, together with other feature extraction algorithms, provides 'radiomic' data, which contain first-, second- and higher-order statistics that quantify the spatial distribution and intensity values of voxels within the tumour $[2,3]$.

Multiple sub-clonal populations of cells are known to coexist within tumours [4]. Texture analysis could act as a 3D surrogate marker of underlying tumour heterogeneity. This, in combination with traditional staging methods, may improve decision tools and optimise treatment pathways [1].

Retrospective studies have investigated the ability of PET texture analysis to predict treatment response and survival in different solid cancers including lung, oesophageal, cervical, and head and neck [5-7]. A large multi-centre study including 1019 patients with lung and head and neck cancer conducted retrospective radiomic analysis on external data sets and demonstrated the additional benefit of CT texture analysis in the staging pathway. Radiomic data were combined with genomic data to produce a prognostic signature resulting in improved prognostic performance compared to traditional Tumour Node Metastasis (TNM) staging alone [1].

This study aimed to demonstrate the additional prognostic value of PET texture analysis compared with the current staging methods by developing a prognostic model in patients with oesophageal cancer (OC). We aimed to calculate a prognostic score that can stratify patients accordingly and perform internal validation of the prognostic model in an independent cohort of patients.

\section{Materials and methods}

\section{Patient cohort}

This is a retrospective cohort study of consecutive patients with biopsy-proven OC, including gastro-oesophageal junctional (GOJ) tumours, radiologically staged between 16 September 2010 and 31 July 2015. All patients were identified at the Regional Upper Gastro-intestinal (GI) Cancer multidisciplinary team (MDT) meeting. Institutional Review Board approval was granted and the requirement for informed consent was waived.

Overall, 550 patients were considered for inclusion. Exclusion criteria were non- or poorly FDG-avid tumours $\left[\operatorname{SUV}_{\max }<3(n=60)\right]$, an MTV $<5 \mathrm{ml}(n=52)$, histology other than adenocarcinoma or squamous cell carcinoma $(n=21)$, a synchronous primary malignancy $(n=7)$ or an oesophageal stent in situ $(n=7)$.
Following exclusions, 403 patients were included and chronologically separated into two independent cohorts. The first (development) cohort included 302 patients radiologically staged between 16 September 2010 and 15 September 2014. The second (validation) cohort included 101 patients radiologically staged between 16 September 2014 and 31 July 2015.

All patients were deemed to have potentially curable disease following contrast-enhanced CT (CECT) staging investigation. All PET/CT examinations were performed separately, following the initial CECT, and reported in the same centre by Consultant Radiologists with an interest in Nuclear Medicine. Clinical, radiological, histological and outcome data were recorded in a prospectively maintained database and were updated in July 2016. Radiological staging was performed according to the Union for International Cancer Control (UICC) TNM 7th edition [8].

\section{PET/CT protocol}

Patients were fasted for at least 6 hours prior to tracer administration. Serum glucose levels were routinely checked and confirmed as less than $7.0 \mathrm{mmol} / \mathrm{L}$ prior to imaging. Patients received a dose of $4 \mathrm{MBq}$ of ${ }^{18} \mathrm{~F}-\mathrm{FDG} / \mathrm{kg}$. Uptake time was 90 min, standard practice at our institution. A GE 690 scanner (GE Healthcare, Buckinghamshire, UK) was used. CT images were acquired in a helical acquisition with a pitch of 0.98 and tube rotation speed of $0.5 \mathrm{~s}$. Tube output was $120 \mathrm{kVp}$ with output modulation between 20 and $200 \mathrm{~mA}$. Matrix size for the $\mathrm{CT}$ acquisition was $512 \times 512$ pixels with a 50 -cm field of view. No oral or intravenous contrast was administered. PET images were acquired at $3 \mathrm{~min}$ per field of view. The length of the axial field of view was $15.7 \mathrm{~cm}$ (skull base to mid-thigh). Images were reconstructed with the ordered subset expectation maximisation algorithm, with 24 subsets and 2 iterations. Matrix size was $256 \times 256$ pixels, using the VUE Point ${ }^{\mathrm{TM}}$ time of flight algorithm.

\section{Treatment protocols}

Patients had surgery alone (SA), neo-adjuvant chemotherapy (NACT) or neo-adjuvant chemoradiotherapy (NACRT) prior to surgery, definitive chemo-radiotherapy (dCRT) or palliative therapy. The optimum treatment strategy was decided by consensus at the MDT. In general, fit patients with tumours preoperatively staged as T3/T4a, N0/N1 were pre-operatively treated with NACT or NACRT. Less fit patients, or those with T1/2 N0 disease, had surgery alone. Patients deemed unsuitable for surgery because of co-morbidity and/or performance status, extensive loco-regional disease or personal choice received dCRT. 


\section{Data preparation and PET segmentation}

Texture analysis of PET images is dependent on the segmentation method used to define the metabolic tumour volume (MTV) [3]. A novel tool called ATLAAS (Automatic Decision Tree Learning Algorithm for Advanced Segmentation) has been developed to standardise segmentation of PET images [9]. Data preparation was performed by a radiology resident (KF) with 4 years' experience of PET research who was blinded to clinical data. ATLAAS segmentation was applied using a graphical user interface (GUI) written in the MatLab language as a plug-in to the Computational Environment for Radiotherapy Research (CERR) [10] (Fig. 1). ATLAAS segmentation first requires creation of a bounding box, which was manually performed in each case. The time taken to perform this process varies, depending on the MTV and proximity to other FDG-avid organs, but can take just a few minutes. Adjustment of the window level and colour of displayed PET images was performed at the discretion of the user, but no pre-defined levels were used since these have no influence on ATLAAS segmentation. The adequacy of ATLAAS segmentation was confirmed by visual assessment in each case. PET images were re-sampled into 0.5 SUV bins. This method is recommended because SUVs are distributed into equally sized intensity bins [11].

\section{Prognostic variables}

Nineteen variables were included in the Cox regression model. Age (number of years) and stage group (I A or B $=1$, II $\mathrm{A}$ or $\mathrm{B}=2$, III A, B or $\mathrm{C}=3, \mathrm{IV}=4$ ) were included. Treatment was divided into curative $(=1)$ and palliative $(=2)$ groups prior to data analysis. $\mathrm{SUV}_{\text {max }}$ and $\mathrm{SUV}_{\text {mean }}$ are the maximum and mean SUV, respectively [12]. MTV derived from ATLAAS and tumour lesion glycolysis (TLG), the product of $\mathrm{SUV}_{\text {mean }}$ and MTV, were calculated [12]. First-order histogram metrics including Histogram Standard Deviation, Histogram Entropy, Histogram Energy, Histogram Skewness and Histogram Kurtosis, were implemented as in Orlhac et al. [5]. Greylevel co-occurrence matrix-based (GLCMs) metrics including Homogeneity, Entropy and Dissimilarity were implemented as in Haralick et al. [13]. Coarseness, implemented as in Amadasun et al. [14], was calculated, along with grey-level size zone matrices (GLSZMs), Intensity Variability, Large Area Emphasis and Zone Percentage, which were implemented as in Thibault et al. [15]. These texture metrics have been selected for inclusion in this study as they have shown prognostic and predictive significance in other texture analysis studies investigating OC [16-18].

\section{Transformation of variables}

Visual inspection of continuous variable histograms was performed before model development to assess for normal distribution and skewness. Specific normality tests were not used but logarithmic transformation of variables with significant long tails was performed prior to analysis to reduce the leverage created from outlying data. Four texture variables were transformed: TLG [log(TLG)], Histogram Energy $[\log ($ Energy $)]$, Coarseness $[\log ($ Coarseness $)]$ and Homogeneity $[\log ($ Homogeneity)]. Repeat inspection of the transformed histograms revealed the four variables had a more normalised distribution.

\section{Metabolic tumour volume and texture metrics}

An important consideration in texture analysis is the range of tumour volumes assessed. Tumours with small volumes may provide redundant texture information because of highly correlated variables [19]. Some authors have suggested excluding tumours with MTV less than $5 \mathrm{ml}$ [5]. Therefore, patients with MTVs $<5 \mathrm{ml}$ were excluded from the analysis.
Fig. 1 Fused axial, sagittal and coronal FDG-PET/CT images of an oesophageal tumour outlined with ATLAAS segmentation tool

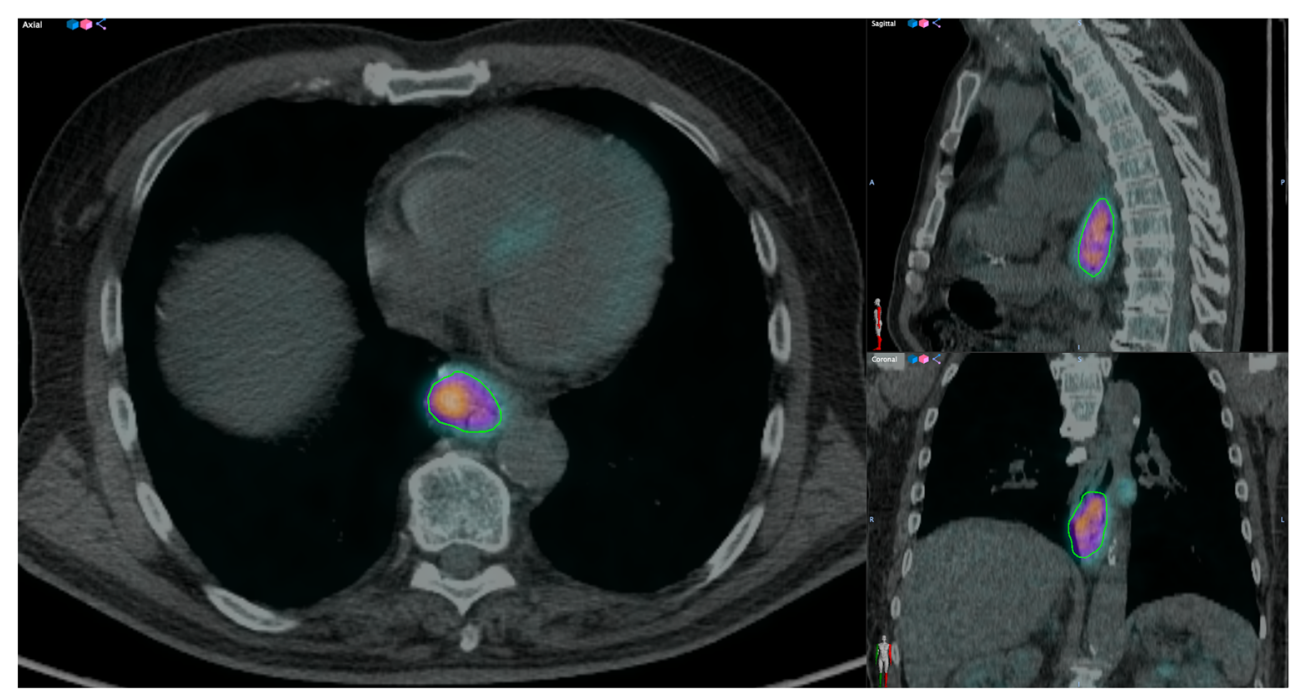




\section{Outcome data}

The primary outcome of the study is OS, defined as number of months survived from date of diagnosis. Patients are followed up 3 monthly for the 1st year, 6 monthly until 5 years then annually thereafter, or until death. All included patients were followed-up for at least 12 months. Date of death was obtained from the Cancer Network Information System Cymru database (CaNISC, Velindre NHS Trust, Wales).

\section{Statistical analysis}

Categorical variables were described as frequency (percent) and continuous variables as median (range) and differences assessed with appropriate nonparametric tests. Cumulative survival was calculated by the Kaplan-Meier life-table method. A Cox Regression Model with a backward conditional method was constructed by an experienced medical statistician. Model power was based on an event-to-variable ratio (EPV), recommended to be a minimum level of 10 [20]. EPV is defined as the ratio of the number of patient deaths compared to the number of variables in the model. The prognostic score was calculated by summation of the products of variables and their corresponding parameter estimate. Using this, patients were separated into quartiles and a log-rank test evaluated significant differences in OS. The effect of curative or palliative treatment on the performance of the prognostic score was assessed with a test of interaction. Furthermore, the Akaike information criterion (AIC) statistic evaluated the estimated quality of three incremental models: (1) a model including age, radiological stage group and treatment; (2) a model including these variables plus newer prognostic indicators $\mathrm{SUV}_{\max }, \mathrm{SUV}_{\text {mean }}$ and MTV; (3) a model including the additional texture metrics. AIC is calculated by $-2 * \log (\mathrm{L})+2 \mathrm{k}$, where $\mathrm{k}$ is the number of parameters and $\mathrm{L}$ is the likelihood of the model [21]. The model with the lowest AIC value is considered the better model. Internal validation of the prognostic model was performed retrospectively in a separate cohort of patients. A $p$-value of $<0.05$ was considered statistically significant. Statistical analysis was performed using SAS version 9.4 (SAS, Cary, NC, USA) and SPSS version 23.0 (IBM, Chicago, IL, USA).

\section{Results}

Baseline characteristics of patients included in the development and validation cohorts are detailed in Table 1. The median OS of the development and validation cohorts was 16.0 months [95\% confidence interval (95\% CI) 13.8-18.2] and 14.0 months (95\% CI 10.4-17.6), respectively. Median follow-up was 43.0 months (95\% CI 35.3-50.7) in the development cohort and 17.0 months (95\% CI 15.7-18.3) in the validation cohort. Overall 1- and 2-year survival in the development cohort was $66.9 \%$ and $33.3 \%$, respectively, and 1 -year OS in the validation cohort was $57.4 \%$. Classification of the radiological EUS and PET/CT TNM stage is detailed in electronic supplementary material, $\mathrm{S} 1$.

\section{Prognostic model development}

The final step of the prognostic model is presented in Table 2. Descriptive statistics for all calculated PET metrics are detailed in electronic supplementary material, S2. There were 232 events and 19 variables in the model, providing 12.2 EPV. In addition to known important prognostic factors in OC (age, radiological stage and treatment), the model identified 3 texture metrics that were independently and significantly associated with survival. The significant variables were $\log$ (TLG), $\log$ (Histogram Energy) and Histogram Kurtosis. Their inclusion in the model illustrates their additional prognostic value compared with current prognostic factors. TLG is calculated as the product of $\mathrm{SUV}_{\text {mean }}$ and MTV [12]. Histogram Energy [5] was calculated using Eq. 1:

Histogram Energy $=\Sigma_{i}(P(i))^{2}$

where $P(i)=\frac{N_{i}}{N}$, with $N_{i}$ the number of voxels of intensity I and $\mathrm{N}$ the total number of voxels. Histogram Kurtosis [5] was calculated using Eq. 2:

Histrogram Kurtosis $=\frac{\frac{1}{N} \Sigma_{i}(I(i)-\mu)^{4}}{\left(\frac{1}{N} \Sigma_{i}(I(i)-\mu)^{2}\right)^{2}}$

where $\mathrm{N}$ is the number of voxels in the image, I(i) is the positive intensity value in the $3 \mathrm{D}$ matrix, and $\mu$ is the mean intensity value.

\section{Prognostic score calculation}

The equation used to calculate the prognostic score in the development cohort was (Stage Group*0.397) $($ Treatment*1.094) $+($ Age*0.024) $-(\log ($ Histogram Energy $) * 1.320)+(\log ($ TLG $) * 1.748)+($ Histogram Kurtosis*0.198). This calculation was derived using published methods [22]. The median score of quartile 1 was -0.73 ( $n=76$, range -1.66 to -0.45 ), quartile 2 was -0.14 ( $n=76,-0.45$ to 0.29$)$, quartile 3 was $0.76(n=75,0.31$ to $1.06)$ and quartile 4 was 1.38 ( $n=75,1.08$ to 2.15$)$. There was a significant difference in OS between quartiles $\left(X^{2} 143.14\right.$, df $3, p<0.001$ ) (Fig. 2). Median OS of quartiles 1 to 4 was 36.0 months (95\% CI 31.1-40.9), 21.0 months (16.1-25.9), 
Table 1 Baseline characteristics of patients in development and validation cohorts

\begin{tabular}{|c|c|c|c|}
\hline Frequency $(\%)$ & Development cohort $(n=302)$ & Validation cohort $(n=101)$ & $p$-value* \\
\hline Median age & 67.0 years (range $39-83$ ) & 69.0 years (range $39-84$ ) & 0.179 \\
\hline Gender (M:F) & 227 (75.2): 75 (24.8) & 78 (77.2): $23(22.8)$ & 0.676 \\
\hline Histology & & & 0.956 \\
\hline Adenocarcinoma & $237(78.5)$ & $79(78.2)$ & \\
\hline Squamous cell Carcinoma & $65(21.5)$ & $22(21.8)$ & \\
\hline Tumour location & & & 0.003 \\
\hline Oesophagus & $192(63.6)$ & $47(46.5)$ & \\
\hline Upper third & $6(3.1)$ & $3(6.4)$ & \\
\hline Middle third & $53(27.6)$ & $10(21.3)$ & \\
\hline Lower third & $133(69.3)$ & $34(72.3)$ & \\
\hline Junction & $110(36.4)$ & $54(53.5)$ & \\
\hline Siewert I & $41(37.3)$ & $24(44.5)$ & \\
\hline Siewert II & $30(27.3)$ & $18(33.3)$ & \\
\hline Siewert III & $39(35.4)$ & $12(22.2)$ & \\
\hline Stage groups & & & 0.238 \\
\hline Stage 1 & $17(5.6)$ & $2(2.0)$ & \\
\hline Stage 2 & $56(18.5)$ & $24(23.8)$ & \\
\hline Stage 3 & $160(53.1)$ & $57(56.4)$ & \\
\hline Stage 4 & $69(22.8)$ & $18(17.8)$ & \\
\hline Treatment & & & 0.624 \\
\hline Curative & $158(52.3)$ & $50(49.5)$ & \\
\hline SA & $24(15.2)$ & $4(8.0)$ & \\
\hline NACT & $67(42.4)$ & $23(46.0)$ & \\
\hline NACRT & $13(8.2)$ & $7(14.0)$ & \\
\hline $\mathrm{dCRT}$ & $54(34.2)$ & $16(32.0)$ & \\
\hline Palliative & $144(47.7)$ & $51(50.5)$ & \\
\hline Overall survival & & & $<0.001$ \\
\hline Alive & $70(23.2)$ & $43(42.6)$ & \\
\hline Dead & $232(76.8)$ & $58(57.4)$ & \\
\hline
\end{tabular}

$S A$ surgery alone; NACT neo-adjuvant chemotherapy; NACRT neo-adjuvant chemoradiotherapy; $d C R T$ definitive chemo-radiotherapy; *chi-square or Mann-Whitney U test
14.0 months (11.7-16.3) and 8.0 months (5.9-10.1), respectively. The interaction test revealed no statistical difference in performance of the prognostic score between curative and palliative treatments $\left(\mathrm{X}^{2} 1.344\right.$, df $\left.1, p=0.246\right)$.

\section{Comparison of estimated model performance}

The AIC of the traditional model including the radiological stage group, treatment and age was 2247.693. The AIC of the
Table 2 Results of the Cox regression model

\begin{tabular}{lccccc}
\hline Prognostic variable & $p$-value & Parameter estimate & Hazard ratio & \multicolumn{2}{c}{$95 \%$ Confidence limits } \\
\cline { 5 - 6 } & & & & Lower & Upper \\
\hline TNM stage & $<0.001$ & 0.397 & 1.49 & 1.20 & 1.84 \\
Treatment & $<0.001$ & -1.094 & 0.34 & 0.24 & 0.47 \\
Age & 0.001 & 0.024 & 1.02 & 1.01 & 1.04 \\
log(Histogram Energy) & 0.011 & -1.320 & 0.27 & 0.10 & 0.74 \\
$\log ($ TLG) & 0.013 & 1.748 & 5.74 & 1.44 & 22.83 \\
Histogram Kurtosis & 0.017 & 0.198 & 1.22 & 1.04 & 1.44 \\
\hline
\end{tabular}


Fig. 2 Kaplan-Meier plot demonstrating overall survival curves of prognostic score quartiles in the development group (X $\mathrm{X}^{2} 143.14$, df 3, $p<0.001)$. Q1 quartile; Q2 quartile 2; Q3 quartile 3; Q4 quartile 4. Median OS of Q1 to Q4 was 36.0 months (95\% CI 31.1-40.9), 21.0 months (16.125.9), 14.0 months (11.7-16.3) and 8.0 months (5.9-10.1), respectively
Patients at Risk, $n$

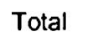

Quartile 1

Quartile 2

Quartile 3

Quartile 4

model that also included $\mathrm{SUV}_{\text {max }}, \mathrm{SUV}_{\text {mean }}$ and MTV was also 2247.693. The AIC of the development prognostic model including additional texture metrics was 2238.007, which was the lowest value. This suggests that incorporation of PET variables and texture metrics improves current prognostic models in $\mathrm{OC}$.

\section{Internal validation of prognostic model}

The prognostic model was applied to the validation cohort. Again, there was a significant difference in OS between patient quartiles $\left(\mathrm{X}^{2} 20.621\right.$, df 3, $\left.p<0.001\right)$ (Fig. 3). Results of PET metrics obtained from the validation cohort are detailed in electronic supplementary material, S3. Mean OS of patients in quartiles 1 and 2 was 16.6 months (95\% CI 13.9-19.3) and 17.4 months (15.4-19.4), respectively. Patients in quartile 1 had lower mean OS than those in quartile 2, but the difference between quartiles was not significant $\left(\mathrm{X}^{2}=0.219, \mathrm{df}=1, p=\right.$ $0.640)$. The median OS for quartiles 3 and 4 was 11.0 months (6.1-15.9) and 9.0 months (4.1-13.9). Three of 26 (11.5\%) patients were treated with palliative intent in quartile 2 , and 2 of $25(8.0 \%)$ patients were treated with curative intent in quartile 3. The AIC of the validation model including PET

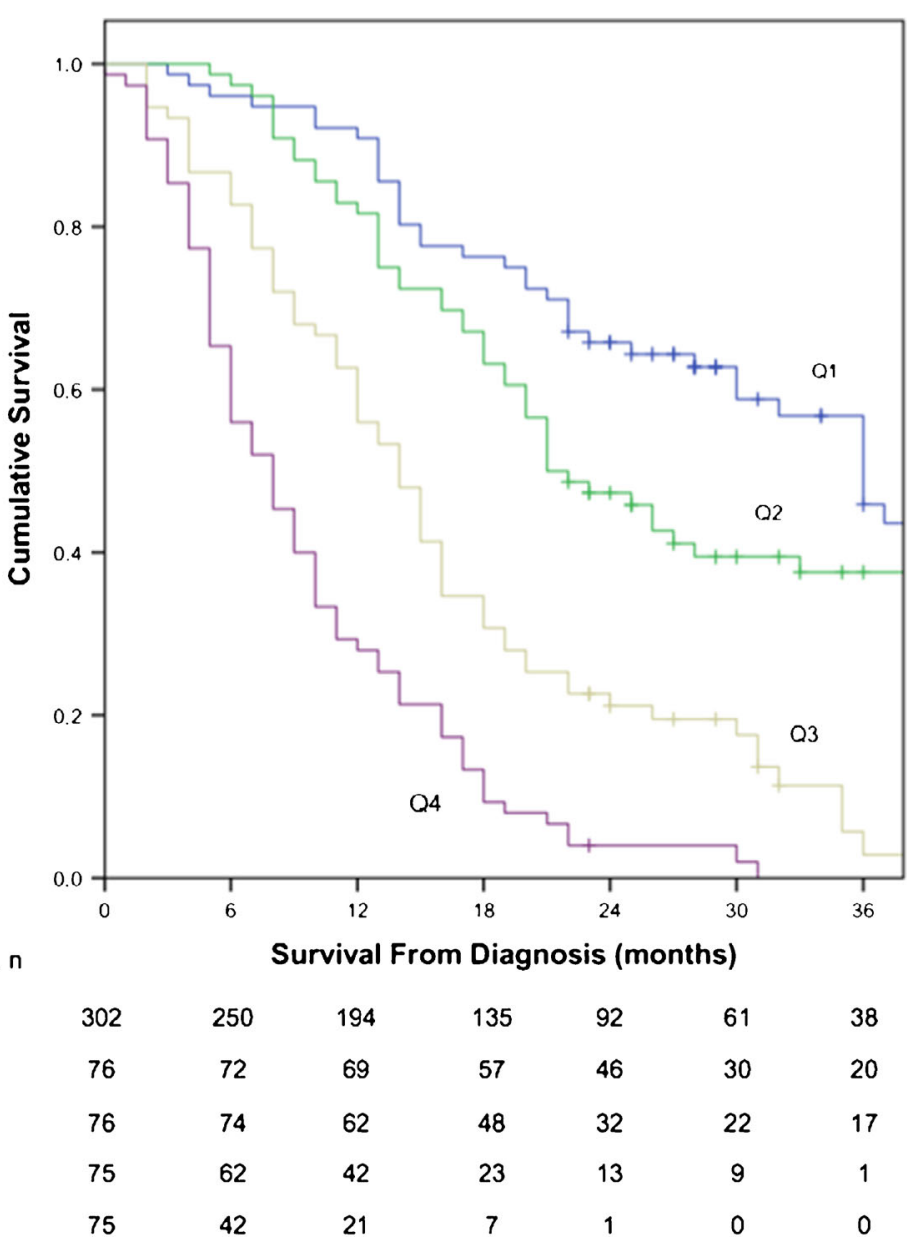

variables and texture metrics was lower (464.671) than in models including the radiological stage group, treatment and age (470.420), and $\mathrm{SUV}_{\text {max }}, \mathrm{SUV}_{\text {mean }}$ and MTV (470.420), respectively.

\section{Discussion}

This study has developed a prognostic model that provides new and important results for OC staging. Internal validation of the model demonstrated a continued difference in OS $(p<0.001)$ between quartiles in an independent cohort of patients. The results of this study show that PET texture analysis may enhance the prognostic TNM staging model in OC.

The prognostic model has identified three PET metrics: $\log$ (TLG), $\log$ (Histogram Energy) and Histogram Kurtosis, which are significantly and independently associated with OS. These metrics have added value over and above currently known prognostic factors: age, radiological stage and treatment. These findings indicate the additional value of novel texture analysis methods in modern staging pathways, which was confirmed with the AIC statistic. Improved riskstratification could identify sub-groups of patients in which a 
Fig. 3 Kaplan-Meier plot demonstrating overall survival curves of prognostic score quartiles in the validation group (X 20.621, df 3, $p<0.001)$. Q1 quartile; Q2 quartile 2; Q3 quartile 3; Q4 quartile 4. Mean OS of patients in Q1 and Q2 was 16.6 months (95\% CI 13.9-19.3) and 17.4 months (15.4-19.4), respectively. Median OS for Q3 and Q4 was 11.0 months (6.115.9) and 9.0 months (4.1-13.9), respectively

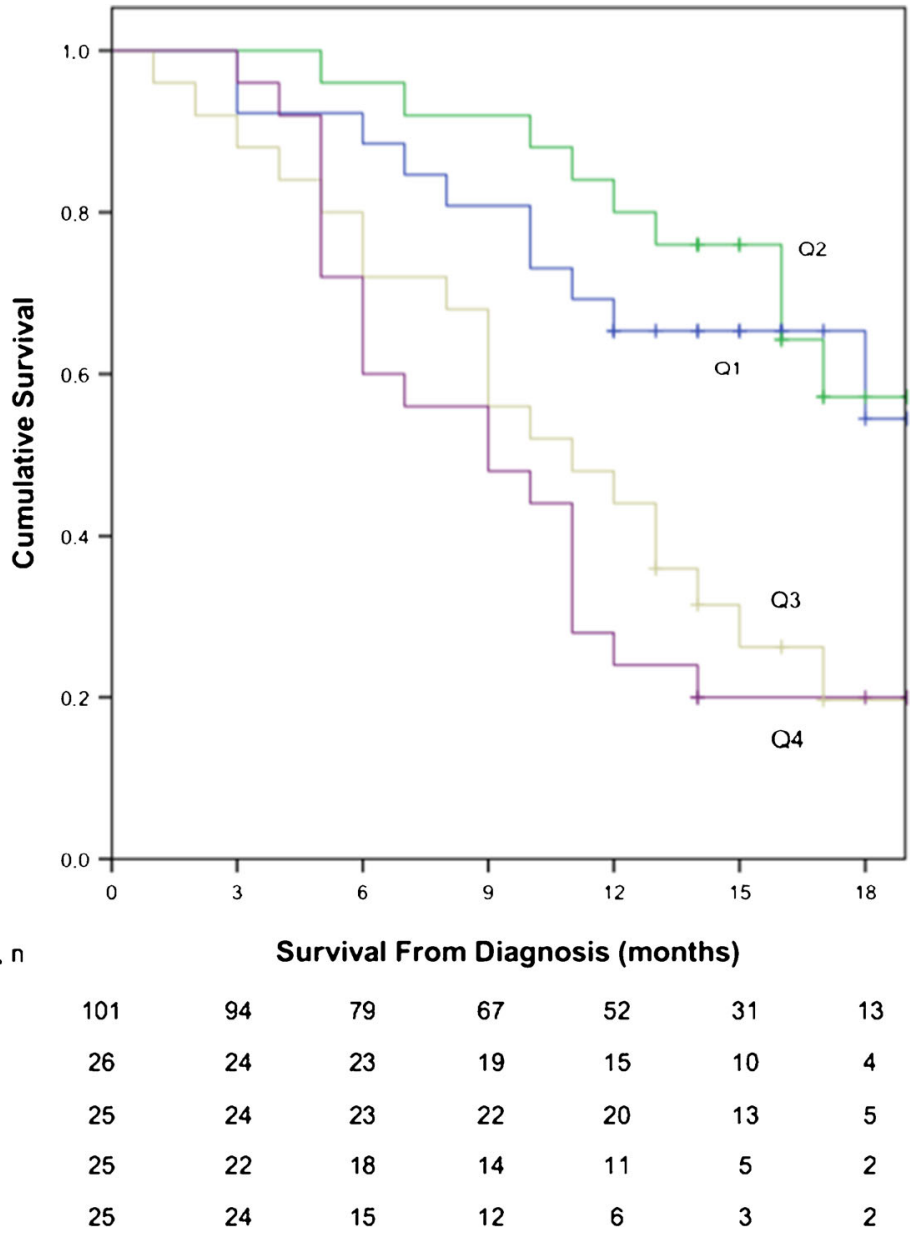

included fewer patients and used different texture analysis software packages.

Texture metrics are dependent on several parameters [28]. The technical implementation of each metric, the segmentation method used, scan acquisition, image smoothing, influence of quantisation and reconstruction parameters all influence the texture analysis results $[3,11,29]$. There are also limitations specific to PET images, given the relatively large voxel volume and presence of noise artefact [30]. Standardisation of texture analysis techniques are essential for multi-centre comparison studies and development of externally validated prognostic models $[11,31]$.

In this study, the texture metrics were derived using the ATLAAS algorithm and a standardised workflow was implemented to ensure reproducible and consistent methods. The benefit of ATLAAS is that the best fitting PET automatic segmentation (PET-AS) method is selected in each individual case from a range of segmentation methods that are built into the ATLAAS algorithm. Commonly used PET-AS methods built into the ATLAAS algorithm include adaptive thresholding, Fuzzy C-means (FCM) and region-growing (RG) methods [9]. ATLAAS was originally designed and tested on patients with FDG-avid head and neck tumours. 
However, it is also applicable to other FDG-avid tumour sites and validation studies are on-going at our institution. Although a new version of ATLAAS had not specifically been designed for this prognostic OC model, visual inspection of the segmented tumour was performed in each case to ensure an appropriate contour had been produced. A benefit of ATLAAS is that tumour segmentation occurs within seconds once the bounding box has been created.

\section{Strengths of study}

This study provides development and internal validation of a prognostic model incorporating PET texture metrics in 403 patients with OC. ATLAAS is a novel machinelearning method that provides robust segmentation results and removes variability by standardising image segmentation. Appropriate statistical methods have been used in this study [23]. The regional upper GI cancer MDT covers a large population of approximately 1.4 million and benefits from the input of highly experienced radiologists, oncologists and surgeons [32].

\section{Limitations of study}

As this study is retrospective, treatment was included in the model and simplified into two groups, curative and palliative. However, the test for interaction showed that the prognostic score could be used in both curative and palliative cohorts with no significant difference in performance. This prognostic model excludes patients with an MTV of less than $5 \mathrm{ml}$ because the quality of the additional data obtained from these models in uncertain [19]. This criterion excludes $11.6 \%$ of potential patients from this study. Another prognostic model including small tumour volumes should be developed for these patients but this model is applicable to many patients with FDG-avid oesophageal tumours.

In conclusion, this large study has developed and validated a prognostic model that demonstrates the additional value of PET texture analysis in OC staging. Three PET metrics, $\log$ (TLG), $\log$ (Histogram Energy) and Histogram Kurtosis, were identified as potentially important variables. These metrics were derived using ATLAAS, a novel machine-learning method designed to optimise and standardise image segmentation. This prognostic model requires further internal and external validation but may be used as a 'benchmark' for further studies investigating the value of PET texture analysis in OC. This study highlights the additional benefit of quantitative imaging techniques in cancer staging, which have the potential to improve patient risk stratification.

Acknowledgements We acknowledge the members of the South East Wales Upper GI cancer MDT.

\section{Compliance with ethical standards}

Guarantor The scientific guarantor of this publication is Dr Ashley Roberts.

Conflict of interest The authors of this manuscript declare no relationships with any companies, whose products or services may be related to the subject matter of the article.

Funding This study has received funding by Tenovus Cancer Care (reference TIG2016/04), Cancer Research Wales (reference 2476) and EPSRC (reference EP/M507842/1) and Velindre NHS Trust (reference 2016/11).

Statistics and biometry Dr Robert Hills kindly provided statistical advice for this manuscript.

Informed consent Written informed consent was waived by the Institutional Review Board.

Ethical approval Institutional Review Board approval was obtained.

\author{
Methodology \\ - retrospective \\ - prognostic study \\ - performed at one institution
}

Open Access This article is distributed under the terms of the Creative Commons Attribution 4.0 International License (http:// creativecommons.org/licenses/by/4.0/), which permits unrestricted use, distribution, and reproduction in any medium, provided you give appropriate credit to the original author(s) and the source, provide a link to the Creative Commons license, and indicate if changes were made.

\section{References}

1. Aerts HJ, Velazquez ER, Leijenaar RT et al (2014) Decoding tumour phenotype by noninvasive imaging using a quantitative radiomics approach. Nat Commun 5:4006

2. Lambin P, Rios-Velazquez E, Leijenaar R et al (2012) Radiomics: extracting more information from medical images using advanced feature analysis. Eur J Cancer 48:441-446

3. Gillies RJ, Kinahan PE, Hricak H (2016) Radiomics: images are more than pictures, they are data. Radiology 278:563-577

4. Gerlinger M, Rowan AJ, Horswell S et al (2012) Intratumor heterogeneity and branched evolution revealed by multiregion sequencing. N Engl J Med 366:883-892

5. Orlhac F, Soussan M, Maisonobe JA, Garcia CA, Vanderlinden B, Buvat I (2014) Tumor texture analysis in 18F-FDG PET: relationships between texture parameters, histogram indices, standardized uptake values, metabolic volumes, and total lesion glycolysis. J Nucl Med 55:414-422

6. Hatt M, Majdoub M, Vallieres M et al (2015) 18F-FDG PET uptake characterization through texture analysis: investigating the complementary nature of heterogeneity and functional tumor volume in a multi-cancer site patient cohort. J Nucl Med 56:38-44

7. van Rossum PS, Fried DV, Zhang L et al (2016) The incremental value of subjective and quantitative assessment of 18F-FDG PET for the prediction of pathologic complete response to preoperative chemoradiotherapy in esophageal cancer. J Nucl Med 57:691-700

8. Sobin LH, Gospodarowicz MK, Wittekind CH (2009) UICC TNM Classification of Malignant Tumours, 7th edn. Wiley, New York 
9. Berthon B, Marshall C, Evans M, Spezi E (2016) ATLAAS: an automatic decision tree-based learning algorithm for advanced image segmentation in positron emission tomography. Phys Med Biol 61:4855-4869

10. Deasy JO, Blanco AI, Clark VH (2003) CERR: a computational environment for radiotherapy research. Med Phys 30:979-985

11. Leijenaar RT, Nalbantov G, Carvalho S et al (2015) The effect of SUV discretization in quantitative FDG-PET radiomics: the need for standardized methodology in tumor texture analysis. Sci Rep 5: 11075

12. Wahl RL, Jacene H, Kasamon Y, Lodge MA (2009) From RECIST to PERCIST: evolving considerations for PET response criteria in solid tumours. J Nucl Med 50:1-50

13. Haralick RM, Shanmugam K, Dinstein I (1973) Textural features for image classification. IEEE Trans Syst, Man Cybern 3:610-621

14. Amadasun M, King R (1989) Textural features corresponding to textural properties. IEEE Trans Syst, Man Cybern 19:1264-1273

15. Thibault G, Fertil B, Navarro C et al. (2009) Texture indexes and gray level size zone matrix application to cell nuclei classification. Pattern Recognit Inf Process: 140-145

16. Tixier F, Le Rest CC, Hatt M et al (2011) Intratumor heterogeneity characterized by textural features on baseline 18F-FDG PET images predicts response to concomitant radiochemotherapy in esophageal cancer. J Nucl Med 52:369-378

17. Yip C, Landau D, Kozarski R et al (2014) Primary esophageal cancer: heterogeneity as potential prognostic biomarker in patients treated with definitive chemotherapy and radiation therapy. Radiology 270:141-148

18. Hatt M, Tixier F, Cheze Le Rest C, Pradier O, Visvikis D (2013) Robustness of intratumour 18F-FDG PET uptake heterogeneity quantification for therapy response prediction in oesophageal carcinoma. Eur J Nucl Med Mol Imaging 40:1662-1671

19. Wu W, Parmar C, Grossmann P et al (2016) Exploratory study to identify radiomics classifiers for lung cancer histology. Front Oncol 6:71

20. Peduzzi P, Concato J, Kemper E, Holford TR, Feinstein AR (1996) A simulation study of the number of events per variable in logistic regression analysis. J Clin Epidemiol 49:1373-1379
21. Akaike H (1974) A new look at the statistical model identification. IEEE Trans Autom Control 19:716-723

22. Moons KG, Kengne AP, Woodward M et al (2012) Risk prediction models: I. Development, internal validation, and assessing the incremental value of a new (bio)marker. Heart 98:683-690

23. Moons KG, Royston P, Vergouwe Y, Grobbee DE, Altman DG (2009) Prognosis and prognostic research: what, why, and how? BMJ 338:b375

24. Blazeby JM, Farndon JR, Donovan J, Alderson D (2000) A prospective longitudinal study examining the quality of life of patients with esophageal carcinoma. Cancer 88:1781-1787

25. Orlhac F, Theze B, Soussan M, Boisgard R, Buvat I (2016) Multiscale texture analysis: from 18F-FDG PET images to histologic images. J Nucl Med 57:1823-1828

26. Yip C, Davnall F, Kozarski R et al (2015) Assessment of changes in tumor heterogeneity following neoadjuvant chemotherapy in primary esophageal cancer. Dis Esophagus 28:172-179

27. Ganeshan B, Skogen K, Pressney I, Coutroubis D, Miles K (2012) Tumour heterogeneity in oesophageal cancer assessed by CT texture analysis: preliminary evidence of an association with tumour metabolism, stage, and survival. Clin Radiol 67:157-164

28. Galavis PE, Hollensen C, Jallow N, Paliwal B, Jeraj R (2010) Variability of textural features in FDG PET images due to different acquisition modes and reconstruction parameters. Acta Oncol 49: 1012-1016

29. Doumou G, Siddique M, Tsoumpas C, Goh V, Cook GJ (2015) The precision of textural analysis in (18)F-FDG-PET scans of oesophageal cancer. Eur Radiol 25:2805-2812

30. Cook GJR, Siddique M, Taylor BP, Yip C, Chicklore S, Goh V (2014) Radiomics in PET: principles and applications. Clin Transl Imaging 2:269-276

31. Lambin P, Zindler J, Vanneste B et al (2015) Modern clinical research: How rapid learning health care and cohort multiple randomised clinical trials complement traditional evidence based medicine. Acta Oncol 54:1289-1300

32. Karran A, Blake P, Chan D et al (2014) Propensity score analysis of oesophageal cancer treatment with surgery or definitive chemoradiotherapy. Br J Surg 101:502-510 